\title{
Method for estimating the cooperativity length in polymers
}

\author{
Marco Pieruccini \\ CNR, Istituto Nanoscienze, v. Campi 213/A, 41125 Modena, Italy
}

Andrea Alessandrini

Dipartimento di Fisica Informatica e Matematica, Università di Modena e Reggio Emilia, v. Campi 213/A, 41125 Modena, Italy

(Received 12 September 2014; revised manuscript received 31 March 2015; published 18 May 2015)

\begin{abstract}
The problem of estimating the size of the cooperatively rearranging regions (CRRs) in supercooled polymeric melts from an analysis of the $\alpha$-process in ordinary relaxation experiments is addressed. The mechanism whereby a CRR changes its configuration is viewed as consisting of two distinct steps: a reduced number of monomers reaches initially an activated state, allowing for some local rearrangement; then, the subsequent regression of the energy fluctuation may take place through the configurational degrees of freedom, thus allowing for further rearrangements on larger length scales. The latter are indeed those to which the well-known Donth's scheme refers. Local readjustments are described in the framework of a canonical formalism on a stationary ensemble of small-scale regions, distributed over all possible energy thresholds for rearrangement. Large-scale configurational changes, instead, are described as spontaneous processes. Two main regimes are envisaged, depending on whether the role played by the configurational degrees of freedom in the regression of the energy fluctuation is significant or not. It is argued that the latter case is related to the occurrence of an Arrhenian dependence of the central relaxation rate. Consistency with Donth's scheme is demonstrated, and data from the literature confirm the agreement of the two methods of analysis when configurational degrees of freedom are relevant for the fluctuation regression. Poly(n-butyl methacrylate) is chosen in order to show how CRR size and temperature fluctuations at rearrangement can be estimated from stress relaxation experiments carried out by means of an atomic force microscopy setup. Cases in which the configurational pathway for regression is significantly hindered are considered. Relaxation in poly(dimethyl siloxane) confined in nanopores is taken as an example to suggest how a more complete view of the effects of configurational constraints would be possible if direct measurements of temperature fluctuations were combined with the proposed analysis.
\end{abstract}

DOI: 10.1103/PhysRevE.91.052603

PACS number(s): 05.20.-y, 61.41.+e, 76.60.-k

\section{INTRODUCTION}

Segmental relaxation in polymers, particularly when approaching the glass transition, is characterized by cooperativity. This is a condition whereby a monomer moves provided the surrounding ones happen to move concurrently. The role of chance here is fundamental and represents an important distinction from other kinds of motion, e.g., those involved in sound propagation.

The existence of an associated length scale of dynamical correlations is inherently addressed by this picture and was put forward already by Adam and Gibbs (AG) in a seminal paper several decades ago, through the introduction of the concept of a cooperatively rearranging region (CRR) [1]. Upon approaching the dynamic glass transition temperature $T_{g}$ from above, this characteristic length would increase. The problem of estimating its limiting value was considered by Donth in a number of papers (see, e.g., [2]), and it was treated extensively in his book [3]. The relevance of this kind of motion, known as the $\alpha$-process, has to do with an understanding of the glass transition.

Evidence of a growing length scale upon decreasing the temperature has been reported in the results of appropriate analyses of the $\alpha$-relaxation patterns observed by, e.g., ordinary dielectric or mechanical spectroscopies. Dielectric response analyzed in terms of either multipoint dynamic correlation functions [4] or Donth's approach [5], and mechanical response analyzed within the framework of a canonical representation of an ensemble of cooperativity regions [6] (related to, but not generally coincident, with the CRRs as explained below), all provide mutually consistent results, albeit from just a qualitative viewpoint. In particular, the average number $z$ of cooperatively rearranging units worked out from the data using the canonical scheme appears to be much smaller than expected for a CRR.

On the contrary, when the same canonical approach is used to analyze relaxation data collected at about the same temperature when a crystallization process of the samples was already driven to completion, a natural thermodynamic criterion for the arrest of crystallization emerges immediately [7].

Further support for this model comes from an analysis of the $\alpha$-relaxation in samples of poly(dimethyl siloxane) (PDMS) confined in nanoporous glasses [8,9]. Reducing the diameter of the pores progressively hinders the glass transition (as indicated by a related lowering of $T_{g}$, accompanied also by a decrease of the specific-heat step $\Delta c_{p}$ ), which disappears at diameters $d_{\text {pore }}=5 \mathrm{~nm}$ and below. A relaxation process following a Vogel-Fulcher-Tammann (VFT) dependence typical of a glassy dynamics is revealed by dielectric spectroscopy for bulk and confined systems down to $d_{\text {pore }}=7.5 \mathrm{~nm}$. This dependence converts to Arrhenian (proper of local modes) for $d_{\text {pore }}=5 \mathrm{~nm}$, and for $d_{\text {pore }}=$ $2.5 \mathrm{~nm}$ the process totally disappears. Upon lowering the temperature and/or $d_{\text {pore }}$, the relaxation broadens in frequency; correspondingly, $z$ increases, as revealed [6] by an analysis carried out on the available data [8]. Rearranging regions in the order of $1 \mathrm{~nm}(z \simeq 8$ at $T=136.6 \mathrm{~K})$ are thus found already at $d_{\text {pore }}=5 \mathrm{~nm}$, so that the absence of relaxation at 
$d_{\text {pore }}=2.5 \mathrm{~nm}$ would not be unexpected. It is worth noting that in the VFT regime, both dielectric and thermal spectroscopies provide quantitatively consistent results for the relaxation rates, that is, the dipole orientation fluctuations and the entropy fluctuations are coupled.

Oriented systems show some peculiarity; for example, the canonical analysis of segmental motion in cold-drawn semicrystalline poly(ethylene terephthalate) (PET) yields values for $z$ [10] that may deviate significantly from those estimated by Donth's method [5].

The above arguments suggest that the cooperative dynamics considered in Donth's theory differs from that revealed by fitting the data using the canonical ensemble scheme (indeed, in $5 \mathrm{~nm}$ confined PDMS, the process still takes place while its glassy character disappears). Notwithstanding the differences, consistencies with the experiments are found with both approaches; therefore, it might be argued that in fact the two pictures reveal different aspects of the same $\alpha$-process.

To be explicit at the outset, the following picture will be proposed. In the temperature interval in which activated configurational motion sets in, i.e., between $T_{g}$ and a higher temperature $T_{A}$ around the melting point $T_{m}$ (if it exists), molecular units move about given locations [11,12], and only after a sufficient fluctuation do they change their configuration cooperatively. This activated (local) rearrangement is a precursor for a subsequent, larger-scale, cooperative motion wherever the energy fluctuation is allowed to regress through diffusive conformational degrees of freedom (the attribute "diffusive" is adopted here as an easy way to refer to the configurational motion on larger length scales, in contrast to the pretransitional, oscillatory motion). The CRRs to which the Donth and AG theories refer are inherent to this large-scale cooperativity. The fluctuation regression is not an activated process, and it may take place also through other paths wherever the diffusive configurational motion is hindered (this may well be the case when relaxation takes place under particular confinement conditions, or when chain orientation is overwhelmingly important).

The scope of this paper is to provide a semiphenomenological model through which CRR sizes can be estimated by a method that differs from that of Donth. It will be shown, however, that the two schemes are indeed very closely related, although not equivalent. The new picture may suggest how the character of the $\alpha$-relaxation can happen to turn "local" when configurational constraints dominate (such as in the $5 \mathrm{~nm}$ confined PDMS). Competition with more fundamental approaches, such as that in Ref. [4], is beyond the scope of this contribution.

\section{A REMINDER ON THE CANONICAL ENSEMBLE APPROACH}

Consider a region consisting of $z$ molecular units in the polymeric melt. This subsystem can be identified by the small cooperativity region responsible for the local relaxation, which can be observed also after the glassy dynamics has been suppressed. At variance from the original formulation [10], the nature of this region differs slightly from that of the CRR introduced by AG; in the present case, indeed, some limited mobility is allowed at its boundaries. Also here, however, all rearranging regions will still be taken to be the same size for simplicity.

Let $\zeta$ be the minimum energy per monomer to be gained by fluctuation for a rearrangement. As described in [10], this threshold depends on the actual configuration of the region, so it fluctuates in time. After this barrier is crossed and a rearrangement initiated, all the energy initially gained is returned to the heat bath (this issue, which was overlooked in the original formulation, is most relevant in the present contribution). Before the transition takes place, the $z$ monomers undergo a collective motion exploring their actual basin of attraction in the phase space. A change of conformation requires that the energy $\langle E\rangle_{\zeta}$, roughly given by averaging on the states above the threshold $\zeta$, is gained by each of these monomers; its product with the probability $w(\zeta)$ that the monomer is in a rearranging state yields approximately the actual amount that is absorbed to reach the top of the barrier and induce a conformational transition. Once this activated state is attained, the configuration may change; however, since the energy threshold of the new configuration is known with just a probability $p(\zeta)$, the entropy $S_{p}=-k_{B} \int d \zeta p(\zeta) \ln p(\zeta)$ must be accounted for in describing this process from a statistical-mechanical point of view. The physical meaning of $p$ is that of a distribution of monomers that are in a mobility state after a barrier height $\zeta$ has been crossed. The entropy $S_{p}$ is thus related to the configurational changes of the (small) cooperativity regions, and it is central to the theory developed in Ref. [10].

The probability distribution $p(\zeta)$ is derived upon extremizing an appropriate potential; this yields

$$
p(\zeta) \sim e^{-\left[w(\zeta)\langle E\rangle_{\zeta}+\lambda \Delta \mu(\zeta)\right] / k_{B} T},
$$

where $k_{B} T$ is the thermal energy and $\lambda$ is a Lagrange multiplier related to the condition that the average $\overline{\Delta \mu} \equiv$ $\int d \zeta p(\zeta) \Delta \mu(\zeta)$ is a constant (in the definition of Ref. [10], this parameter encompassed the term $\left.k_{B} T\right) ; \Delta \mu(\zeta)=$ $-k_{B} T \ln w(\zeta)$ is the rearrangement chemical potential given in terms of the probability,

$$
w(\zeta) \equiv \frac{Z_{\zeta, n}}{Z_{0, n}}
$$

which in turn is expressed through the partition function

$$
Z_{\zeta, n} \equiv \int_{\zeta}^{\infty} d \epsilon \epsilon^{n} e^{-\epsilon / k_{B} T} .
$$

Note that, apart from a factor that is irrelevant presently, $Z_{0, n}$ is the partition function of an ensemble of $n+1$ independent oscillators [13].

The meaning of Eq. (1) is simple: The subset of (mobile) monomers with given $\zeta$ is larger the lower the collective rearrangement free energy $\lambda \Delta \mu(\zeta)$ is; on the other hand, the absorption of mean energy $w(\zeta)\langle E\rangle_{\zeta}$ promotes the transition to different configurations, i.e., to states with different $\zeta$ value, thus depleting the same subset.

Figure 1 shows the $\zeta$ dependence of (i) the average energy $\langle E\rangle_{\zeta}$, and (ii) its product with the probability $w(\zeta)$, which can be expressed in terms of the incomplete $\Gamma$ function as

$$
w\langle E\rangle_{\zeta}=k_{B} T(n+1) \frac{\Gamma\left(n+2, \zeta / k_{B} T\right)}{\Gamma(n+2)} .
$$




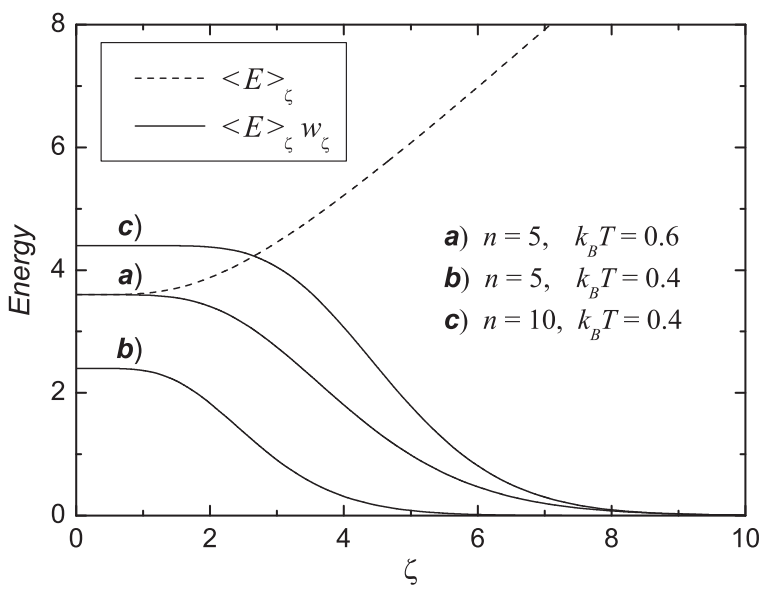

FIG. 1. Average energy $\langle E\rangle_{\zeta}$ and its product with $w_{\zeta}$ as functions of the energy threshold $\zeta$. All energies are expressed in $\mathrm{kcal} / \mathrm{mol}$.

Once $k_{B} T$ and $\zeta$ are assigned, a region can rearrange at a significant rate provided $n$ is large so as to make $w\langle E\rangle_{\zeta}>0$ sufficiently. Upon increasing $\zeta, n$ must also be incremented to maintain mobility. If the temperature decreases, on the other hand, the upper bound below which $w\langle E\rangle_{\zeta}$ is non-negligible shifts toward lower $\zeta$ values and mobility is recovered once again assigning larger values to $n$. Upon fitting the data, $n$ is always found to increase with the number $z$ of units in a rearranging region, so the meaning of this argument is that the average size of the rearranging regions increases when either $T$ lowers at given $\zeta$ or the latter increases (by, e.g., cross-links or crystal confinement) at fixed $T$. In other words, mobility characterizes those regions for which $\overline{\Delta \mu}$ is appropriately small, and this requires that they be large enough or $\zeta$ is low.

The relaxation function is an average over the distribution $p(\zeta)$ of single-time decays [10]:

$$
\phi(t) \sim \int_{0}^{\infty} d \zeta w^{\lambda} e^{-w\langle E\rangle_{\zeta}} e^{-t / \tau(\zeta)}
$$

where

$$
\tau(\zeta) \equiv \tau^{*} e^{z \Delta \mu(\zeta) / k_{B} T}
$$

is the actual relaxation time of the $z$ monomers in a configuration characterized by a barrier $\zeta$, and the identity $w^{\lambda}=\exp \left\{-\lambda \Delta \mu / k_{B} T\right\}$ has been used. Upon fitting the data, one expects (as is indeed found) that $\lambda$ will remain close to $z$ (i.e., within the same order of magnitude); thus, the Lagrange multiplier associated with the condition $\overline{\Delta \mu}=$ const during the transitions between configurations with different $\zeta$ is related to the number of units in the rearranging region.

\section{CRR SIZE}

As anticipated in the Introduction, the onset of a large-scale cooperative rearrangement is viewed here as a possible path through which an energy fluctuation such as those considered above regresses. There are at least two aspects that should be taken into account regarding the relevance of this mechanism. On the one hand, a small enough specific-heat step $\Delta c_{p}$ at $T_{g}$ should guarantee that the energy released by the activated $z$ monomers rapidly spreads out over a larger domain on the way back to the heat bath. On the other, the coupling to the diffusive configurational degrees of freedom should be efficient, which is expected indeed, since both the small- and large-scale motions considered relate to the same degrees of freedom.

\section{A. Rearrangement free energy versus configurational entropy}

As a preliminary step, it is worth considering some known experimental results and their analysis with the canonical ensemble model. For convenience, the system to be considered specifically is PET.

Scanning temperature dynamic mechanical spectroscopy on an initially glassy PET sample shows a relatively narrow $\alpha$-process with a $\tan \delta$ peak centered at $T \approx 85^{\circ} \mathrm{C}$ (with a probing frequency of $0.3 \mathrm{~Hz}$ and a scanning rate of $2^{\circ} \mathrm{C} / \mathrm{min}$ ) [14]. After cold crystallization at $T_{c}=100^{\circ} \mathrm{C}$, followed by an annealing at that temperature for an overall period of $7 \mathrm{~h}$, a similar analysis carried out under the same experimental conditions reveals a broader $\alpha$ peak centered at $T \approx 100^{\circ} \mathrm{C}[15]$. This is an effect of the dynamical constraints introduced by crystal formation.

Isothermal mechanical spectroscopy carried out on a semicrystalline PET sample, prepared as before from the same raw material, revealed a segmental relaxation process consisting of two components (see also [16]): a slow and broad one, and a fast one localized in amorphous regions where dynamic constraints seem to be less effective. The analysis was carried out in the $10^{-3}-60 \mathrm{~Hz}$ frequency range for temperatures of 85,90 , and $95^{\circ} \mathrm{C}$ (for each temperature, the frequency scan was repeated twice, showing no significant change in the loss pattern). Details can be found in Ref. [17], but the relevant outcomes for the present discussion are the fast mode $\overline{\Delta \mu}$ values worked out by means of the canonical ensemble scheme and reported in Table I for easy reference (the data of Ref. [17] have been reanalyzed here with better integration routines).

The central relaxation times of the fast component have been fitted with the VFT function

$$
\tau_{0}=\tau_{\infty} e^{D T_{\mathrm{VFT}} /\left(T-T_{\mathrm{VFT}}\right)},
$$

with $D$ the fragility parameter and $T_{\mathrm{VFT}}$ the Vogel temperature. The latter has been estimated by setting $\tau_{\infty}$ equal to $10^{-14} \mathrm{~s}$ (a value that turns out to be appropriate for a number of systems [18]). This yields $T_{\mathrm{VFT}}=318.7 \mathrm{~K}$, which is in good agreement with independent analyses (cf., e.g., [5]). Of course, this value cannot be considered a precise determination of $T_{\mathrm{VFT}}$ but merely a fitting number, as it was worked out from just

TABLE I. Rearrangement chemical potential $\overline{\Delta \mu}$ and configurational entropy per monomer $s_{c}$, times $T$, for semicrystalline PET (both in $\mathrm{kcal} / \mathrm{mol}$ ) at different temperatures (in ${ }^{\circ} \mathrm{C}$ ), calculated for a Kauzmann temperature $T_{K} \simeq 319 \mathrm{~K}$ and a specific-heat step per monomer $\Delta c_{p} \approx 9.3 k_{B}$ at $T_{g}$.

\begin{tabular}{lll}
\hline \hline$T$ & $\overline{\Delta \mu}$ & $T s_{c}$ \\
\hline 85 & 0.67 & 0.76 \\
90 & 1.00 & 0.86 \\
95 & 1.36 & 0.97 \\
\hline \hline
\end{tabular}


three data points. By the way, since it is to be used in place of the Kauzmann temperature $T_{K}$ for an estimate of the specific configurational entropy

$$
s_{c}=\Delta c_{p} \ln \left(\frac{T}{T_{K}}\right)
$$

and in this case $T-T_{K} \gtrsim 40 \mathrm{~K}$ and $T \gtrsim 360 \mathrm{~K}$ (so that $\left|\delta s_{c} / s_{c}\right| \sim 10 \delta T_{K} / T_{K}$ ), its exact determination is not necessary.

The product $T s_{c}$ is reported in Table I as well, and it can be readily noticed that the rearrangement chemical potential $\overline{\Delta \mu}$ is larger than $T s_{c}$ except for $T$ close to the lowest value. Given the above results from dynamic mechanical spectroscopy, and being that $T_{g} \approx 75^{\circ} \mathrm{C}$ for amorphous PET, a value of $85^{\circ} \mathrm{C}$ can be considered very close to the glass transition temperature within the higher mobility regions of the crystallized sample (in fact, $T_{g} \simeq 80^{\circ} \mathrm{C}$ within these domains seems reasonable, as indicated by the calorimetric analysis in Ref. [14]).

The data listed in the table suggest that the approximate relation

$$
\frac{\overline{\Delta \mu}}{T} \gtrsim s_{c}
$$

(to be implemented below in a more appropriate form) may relate to the actual criterion for the onset of large-scale mobility. The configurational entropy would play the role of a rearrangement threshold. Wherever small-scale fluctuations below $T_{g}$ occur, they would be unable to excite significant diffusive motions upon regression, so the energy would return to the heat bath through different paths, with a structural arrest hanging over.

The meaning of Eq. (9) becomes clear if it is reexpressed in terms of probabilities. Let $\zeta^{*}$ be a characteristic value of the energy threshold for which $\overline{\Delta \mu}=-k_{B} T \ln \left(Z_{\zeta^{*}, n} / Z_{0, n}\right)$, and let $\Omega_{c}$ be the number of (low-energy) configurational states counted on a per monomer basis, such that $\Omega_{c} \sim e^{s_{c} / k_{B}}$. Then, Eq. (9) reads

$$
\Omega_{c}^{-1} \gtrsim \frac{Z_{\zeta^{*}, n}}{Z_{0, n}},
$$

that is, the probability associated with the final state is larger than that of the initial activated state; in other words, the fluctuation regression is spontaneous.

Wherever the energy needed to induce a configurational change is too large (e.g., possibly, in the $5 \mathrm{~nm}$ confined PDMS of the Introduction), there is no way to get it from the activated state; the fluctuation energy returns to the heat bath through other mechanisms, and the number of rearranging units remains $z$. The process, then, is (almost) local.

\section{B. Large-scale cooperativity}

Based on the hint given by Eq. (9), it is assumed as a working hypothesis that all the energy of the activated regions for which $\Delta \mu(\zeta)>T s_{c}$ is completely delivered to the diffusive conformational degrees of freedom when the fluctuation regresses (this models the energy transfer efficiency considered at the beginning of the section). This energy induces, on a number $N_{\text {ind }}$ of surrounding monomers, actual configurational motion with an associated entropy of $N_{\text {ind }} s_{c}$.
Consistently with an efficiency that is assumed high, the energy transfer to the $N_{\text {ind }}$ monomers is considered to be approximately reversible, yielding

$$
N_{\text {ind }} \approx z \frac{\kappa \bar{\zeta}}{T s_{c}},
$$

where

$$
\kappa \bar{\zeta}=\frac{1}{M} \int_{\zeta_{0}}^{\infty} d \zeta \zeta p(\zeta)
$$

is the product between the fraction $\kappa=M^{-1} \int_{\zeta_{0}}^{\infty} d \zeta p(\zeta)$ of mobile regions with $\Delta \mu(\zeta)>s_{c}$, and the average energy associated with each of its $z$ monomers; $M \equiv \int_{0}^{\infty} d \zeta p(\zeta)$ is the normalization constant for $p$, and $\zeta_{0}$ is such that $\Delta \mu\left(\zeta_{0}\right)=T s_{c}$. Then, the total number of monomers that actually rearrange cooperatively is given by

$$
N_{\alpha}=N_{\text {ind }}+z
$$

As can be imagined, Eq. (9) does not have to be taken in a strict sense as a condition for large-scale rearrangement, since it is indeed an indicative criterion involving averages. The relevant quantities are in fact the mean energy $\bar{\zeta}$ of the fraction $\kappa$ of monomers above the threshold, and these may be significant also in situations in which Eq. (9) is not, or marginally is not, fulfilled.

\section{RELATION WITH DONTH'S APPROACH}

To better point out its relationship with the model developed in the previous section, a simple derivation of Donth's formula for the estimate of the CRR size will be given below. Donth's reasonings leading to this formula are complex, and not at all trivial [3], so the arguments below must be considered as just a naïve picture to provide a framework for a discussion.

\section{A. Cooperativity}

The problem of cooperative rearrangements at the glass transition is treated by Donth in the framework of a fluctuation theory $[2,3]$. In this respect, the approach adopted by Landau to relate the entropy fluctuations with the associated minimal work (Secs. 20 and 112 of Ref. [19]) is central: A subsystem is allowed to fluctuate while maintaining a state of internal thermodynamic equilibrium; the dependence of its mean entropy $S$ on the other thermodynamic variables is thus defined. Of course, the assumption of internal equilibrium for the subsystem allows for internal fluctuations as well.

The CRR can be grossly partitioned in two subsystems: one encompassing all the diffusional configurational degrees of freedom, and the other collecting the remainder. Between these two parts, energy exchanges occur as a manifestation of CRR internal fluctuations. Let $c_{p}$ be the specific heat of the nondiffusional degrees of freedom (as in [2], $c_{p} \approx c_{v}$ will be understood; moreover, we assume that $\Delta c_{p} \ll c_{p}$ ). The meansquare amplitude of the entropy fluctuations $\overline{\delta S^{2}}$ undergone by the CRR as a whole is linear in the derivative $\partial S / \partial T$ :

$$
\overline{\delta S^{2}}=k_{B} T \frac{\partial S}{\partial T},
$$


where on the right-hand side $\partial S / \partial T=N c_{p} / T$, with $N$ the number of monomers in the CRR. The set of configurational degrees of freedom of the CRR is not always (and often not totally) involved in each of these fluctuations; however, the linearity of the right-hand side in the preceding equation allows us to estimate the average mean-square fluctuation of the configurational entropy as $\overline{\delta S_{c}^{2}} \sim \overline{\delta S^{2}}\left[\Delta c_{p} / c_{p}\right]$. One may imagine an intermittent variation in $S_{c}$, with a rearrangement taking place at each entropy burst [this is indeed the same mechanism underlying Eq. (11)]. Every arrest of the diffusional motion is related to a transfer of energy $\delta E_{c}$ from the configurational to the nonconfigurational part of the CRR; in turn, the latter undergoes temperature fluctuations with an average amplitude $\delta T \sim \delta E_{c} /\left(N c_{p}\right)$. Thus

$$
\overline{\delta T^{2}} \approx\left(\frac{\partial S}{\partial T}\right)^{-2} \overline{\delta S_{c}^{2}} \simeq \frac{k_{B} T^{2}}{N} \Delta\left(\frac{1}{c_{p}}\right),
$$

where $\Delta\left(1 / c_{p}\right)$ is the change of the inverse specific heat across $T_{g}$. This is Donth's formula, relating the cooperativity $N$ with the temperature fluctuations observable at $T_{g}$ [2].

Equation (11) describes the mechanism whereby a nonzero $S_{c}=N_{\text {ind }} S_{c}$ is maintained by the regression of energy fluctuations. On the other hand, a "dissipation" of $S_{c}$ via the energy transfer toward the nonconfigurational degrees of freedom is contained in Eq. (15). The latter equation, in fact, provides $\overline{\delta T^{2}}$ as a consequence of a steady back and forth energy exchange between the two subsystems in which the CRR is partitioned, so one can say that indeed Eq. (11) is another way to reexpress [of Eq. (15)] just the mechanism of $S_{c}$ gain.

To be more explicit, we can find the average entropy $S_{c}$ by integrating a fluctuation-dissipation relation such as Eq. (14) [which is in fact the r.h.s. equality of Eq. (15)] for the subsystem consisting of just the configurational degrees of freedom:

$$
S_{c}=\int_{T_{K}}^{T} d \theta \frac{\overline{\delta S_{c}^{2}}}{k_{B} \theta}=N s_{c},
$$

where the $T$ dependence of $\Delta c_{p}$ has been neglected and Eq. (8) has been used. Then, it follows immediately that

$$
N \equiv N_{\text {ind }},
$$

and the consistency between the results derived by Donth's method and the present one is indirect proof that Eq. (11) correctly matches the energy transfer toward the configurational degrees of freedom included in Eq. (15).

The correspondence expressed by Eq. (17) is important because it may help to construct a more comprehensive view about how confinement, in actual situations, affects configurational motion. With regard to small-scale rearrangements, the canonical description is able to reveal the presence of dynamical constraints (see, e.g., [6] and some of the analyses below); the large-scale cooperativity instead, i.e., $N_{\text {ind }}$, can only be estimated through Eq. (11) assuming that Eq. (8) holds, and after some values for $\Delta c_{p}$ and $T_{K}$ are assigned. Although the latter can be estimated from the VFT behavior, the appropriate $\Delta c_{p}$ in the presence of constraints may not be unambiguously accessible (see, e.g., the case of PDMS in Ref. [8]). The condition expressed by Eq. (17) (or an equivalent one) thus may help us to gain a deeper insight into the problem, at least in principle, for example by comparing the expected temperature fluctuations (calculated as shown below) with the measured ones.

\section{B. Temperature fluctuations}

The temperature changes induced in the configurational part of the CRR by the fluctuation regression can be approximately estimated from the direct analysis of the relaxation data as follows. We focus on a set of $z$ monomers that may form a small-scale rearranging domain upon sufficient fluctuation. On average, these $z$ units are in a stationary state characterized by a chemical potential $\overline{\Delta \mu}$; depleting the dynamical constraints, the rearrangement free energy $F \equiv z \overline{\Delta \mu}$ would decrease and the system would approach a higher entropy state (of thermodynamic equilibrium at most). In other words, the stationary (metastable) state of these units is characterized by minimal work with respect to the equilibrium state [19]; let $R_{0}=F$ be this minimal work. Large-scale rearrangements require that free-energy fluctuations $z \Delta \mu(\zeta)>z T s_{c}$ occur; the minimal work characterizing the states precursory to rearrangement, $\left.R_{p} \equiv z \overline{\Delta \mu}\right|_{\zeta>\zeta_{0}}$, results from an average over $p(\zeta)$. Due to the existence of the threshold $s_{c}>0$, it is always $R_{p}>R_{0}$

Once the precursory state is attained, a rearrangement takes place with a probability $\kappa$; in this case, configurational constraints drop to some extent because the large-scale motion begins. The system moves toward higher entropy states, and in doing so it may overshoot the initial state characterized by $R_{0}$, which can then be reached again after the rearrangement has occurred.

In steady conditions, the energy delivered to the configurational part of the CRR equals $W=\kappa\left(R_{p}-R_{0}\right)$, and one finds immediately (cf. [19], Sec. 20)

$$
\Delta T \sim \frac{W}{S_{c}}=\frac{T}{\bar{\zeta}}\left[\left.\overline{\Delta \mu}\right|_{\zeta>\zeta_{0}}-\overline{\Delta \mu}\right] .
$$

The fluctuation-dissipation relation for $S_{c}$ [cf. Eq. (14) for $S]$ implies that $\overline{\delta T \delta S_{c}}=k_{B} T$. For this reason, one expects that $W \approx k_{B} T$, and this is always found indeed when analyzing the data.

\section{ANALYSIS OF EXPERIMENTAL RESULTS}

The relaxation function of the $\alpha$-process is central to the evaluation of the cooperativity with the method proposed in this paper. Often, dielectric or mechanical spectroscopies are used to extract this function in the time interval of interest. More recently, a method based on an appropriate analysis of the free induction decay echoes in ${ }^{1} \mathrm{H}$-NMR relaxometry of systems dominated by dipolar interaction was proposed and tested on polybutadiene [20].

For responses resolved in frequency, one usually starts from the Havriliak-Negami (HN) analysis of, e.g., mechanical or dielectric losses worked out in isothermal conditions for a range that is as wide as possible. Details can be found, for instance, in Refs. [6,10,17]. Once the HN parameters for the process of interest have been found, the corresponding relaxation function $\phi_{\mathrm{HN}}(t)$ is calculated by cosine Fourier 
transforming:

$$
\phi_{\mathrm{HN}}(t)=\frac{2}{\pi} \int_{0}^{\infty} \frac{A^{\prime \prime}\left(\omega ; a, b, \tau_{0}\right)}{\Delta A} \cos (\omega t) \frac{d \omega}{\omega},
$$

where $A^{\prime \prime}$ is the imaginary part of the complex response function (i.e., either the dielectric permittivity or the mechanical modulus) expressed in the form

$$
A=\frac{\Delta A}{\left[1+\left(i \omega \tau_{0}\right)^{a}\right]^{b}},
$$

with $a$ and $b$ the width and asymmetry parameters $(a, b \leqslant 1$; $b=1$ for a symmetric process) and $\tau_{0}$ the central relaxation time.

The analysis then proceeds by adjusting Eq. (5) on the experimental relaxation function $\phi_{\mathrm{HN}}(t)$. The fitting parameters are $\lambda, z$, and $\tau^{*}$, while the exponent $n$ is chosen in order to reach a minimum $\chi^{2}$ with the prescription that the lower limit $t_{\text {min }}$ of the fitting interval is pushed toward low $t$ values so as to keep it close to $\tau^{*}$.

With this procedure, the average rearrangement chemical potential $\overline{\Delta \mu}$, the average height of the energy threshold $\langle\zeta\rangle$, and all other ingredients needed to calculate $N_{\alpha}$ can be derived. $\Delta c_{p}$ is obtained by calorimetry and $T_{K}$ is identified with $T_{\mathrm{VFT}}$ after $\tau_{0}(T)$ [Eq. (7)] has been adjusted to the data.

Upon each fitting, it is always found that $\lambda$ and $z$ remain within the same order of magnitude; moreover, it is also found that

$$
\tau_{0} \approx \tau^{*} e^{z \overline{\Delta \mu} / k_{B} T}
$$

The fulfillment of these conditions reflects a mutual dependence of the fitting parameters, and it represents an effective reduction of their number.

\section{A. Unoriented $100{ }^{\circ} \mathrm{C}$ crystallized PET}

The dynamic mechanical analysis of a sample coldcrystallized at $T_{c}=100^{\circ} \mathrm{C}$ and annealed at the same temperature for an overall duration of $7 \mathrm{~h}$ yields the loss patterns of Fig. 2 relative to temperatures of 85,90 , and $95^{\circ} \mathrm{C}$.

For $T=90$ and $95^{\circ} \mathrm{C}$, it is possible to separate the fast and slow contributions as shown in the figure (at $85^{\circ} \mathrm{C}$, the slow component cannot be revealed anymore in the observable frequency interval). It is known that, in general, the presence of the crystals symmetrizes the frequency profile of the relaxation, so that $b=1$ is set for both the fast and slow components when they have to be separated out of the overall

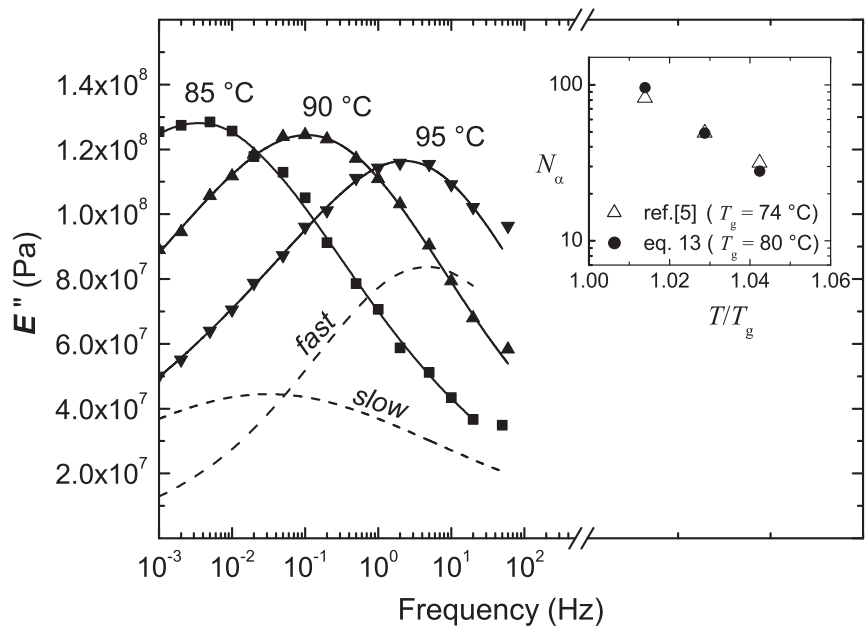

FIG. 2. Mechanical loss patterns of a PET sample coldcrystallized at $T_{c}=100^{\circ} \mathrm{C}$ for $7 \mathrm{~h}$. The dashed lines represent the fast and slow components deconvoluted from the $T=95^{\circ} \mathrm{C}$ curve. The inset shows a comparison of the $N_{\alpha}$ estimates based on Donth's method [5] (open triangles) and the present analysis (filled circles).

pattern. The result is consistent with independent analyses of dielectric response (cf., e.g., [16]).

For a comparison with previous analyses carried out under the scheme of Donth, just the fast mode relaxation can be considered. Contributions to the entropy fluctuations arising from the slow mode are also present, but they are difficult to estimate because of the lack of an available, sufficiently wide analysis of its temperature dependence (e.g., to get an appropriate $T_{K}$ for it). In any case, this effect is expected to be small, as can be argued, e.g., from the relative amplitudes of the two modes, with the fast one overwhelming the other, particularly at low temperatures.

Table II collects the results of the fitting procedure to the responses of Fig. 2 (the fact that thermodynamically small regions are being observed is pointed out by the relation $\overline{\Delta \mu} \sim \delta[\Delta \mu][10]$ resulting from the fittings). Note that previously published analyses of the same data [6,17] only provided small-scale cooperativities, $z$, whereas in the present case $N_{\alpha}, \kappa$, and $\Delta T$ are also obtained.

A comparison with the estimates given in [5] on the basis of Donth's theory is shown in the inset of Fig. 2, where $T_{g}=$ $80^{\circ} \mathrm{C}$ has been assumed for the amorphous regions of PET where the fast mode is localized.

TABLE II. Temperature $T$, HN parameters $a$ and $\tau_{0}\left(b \equiv 1\right.$, see text), fitting parameters $\lambda, z, \tau^{*}$ (and $n$ ), lower limit of the fitting interval $t_{\min }$, and resulting values for the average energy threshold $\langle\zeta\rangle$, the average excess energy above the threshold $\bar{\zeta}$ [cf. Eq. (12)], the rearrangement chemical potential $\overline{\Delta \mu}$ and its dispersion around the mean $\delta[\Delta \mu]$, the fraction $\kappa$ of CRRs inducing diffusive configurational changes, the

\begin{tabular}{|c|c|c|c|c|c|c|c|c|c|c|c|c|c|c|}
\hline $\begin{array}{l}T \\
\left({ }^{\circ} \mathrm{C}\right)\end{array}$ & $a$ & $\begin{array}{l}\tau_{0} \\
(\mathrm{~s})\end{array}$ & $n$ & $\lambda$ & $z$ & $\begin{array}{l}\tau^{*} \\
(\mathrm{~s})\end{array}$ & $\begin{array}{l}t_{\min } \\
(\mathrm{s})\end{array}$ & $\begin{array}{c}\langle\zeta\rangle \\
\left(\frac{\mathrm{kcal}}{\mathrm{mol}}\right)\end{array}$ & $\begin{array}{c}\bar{\zeta} \\
\left(\frac{\mathrm{kcal}}{\mathrm{mol}}\right)\end{array}$ & $\begin{array}{c}\overline{\Delta \mu} \\
\left(\frac{\mathrm{kcal}}{\mathrm{mol}}\right)\end{array}$ & $\begin{array}{c}\delta[\Delta \mu] \\
\left(\frac{\mathrm{kcal}}{\mathrm{mol}}\right)\end{array}$ & $\kappa$ & $N_{\alpha}$ & $\begin{array}{c}\Delta T \\
\left({ }^{\circ} \mathrm{C}\right)\end{array}$ \\
\hline 85 & 0.31 & 50 & 18 & 9.8 & 9.8 & $2.3 \times 10^{-3}$ & $2.6 \times 10^{-3}$ & 13.4 & 15.5 & 0.67 & 0.36 & 0.39 & 96 & 8.1 \\
\hline 90 & 0.34 & 0.64 & 10 & 4 & 5.8 & $1.2 \times 10^{-4}$ & $2.4 \times 10^{-4}$ & 9.1 & 10.4 & 1 & 0.56 & 0.63 & 49 & 11.6 \\
\hline 95 & 0.37 & 0.035 & 7 & 2.3 & 3.9 & $2.5 \times 10^{-5}$ & $2.6 \times 10^{-5}$ & 7.7 & 8.6 & 1.36 & 0.76 & 0.75 & 28 & 16 \\
\hline
\end{tabular}
total number of rearranging monomers $N_{\alpha}$, and the approximate temperature fluctuation $\Delta T$ for the fast relaxation process of the $T_{c}=100^{\circ} \mathrm{C}$ cold-crystallized PET sample at the temperatures of 85,90 , and $95^{\circ} \mathrm{C}$. 
Evaluating the CRR diameter with the expression

$$
\xi \sim \sqrt[3]{\frac{\mathrm{mw} N_{\alpha}}{\rho N_{A}}},
$$

with mw the molecular weight of the monomer, $\rho$ the density, and $N_{A}$ the Avogadro number, one finds it to be around 3, 2.4, and $1.9 \mathrm{~nm}$, respectively, at 85,90 , and $95^{\circ} \mathrm{C}$.

With regard to the temperature fluctuations, Eq. (18) yields the estimates listed in the last column of Table II; linear regression gives $\Delta T \simeq 4{ }^{\circ} \mathrm{C}$ at a temperature of $80^{\circ} \mathrm{C}$. This result can be considered in good agreement with Ref. [5], which reports $\Delta T \simeq 3.1^{\circ} \mathrm{C}$ at $T_{g}$ in the absence of crystals.

\section{B. Oriented PET}

The analysis of cold-drawn PET (with a drawing ratio of 4), subsequently crystallized at $140^{\circ} \mathrm{C}$, yields a number of rearranging units (at the small scale) of $z \simeq 7$ at $T=$ $130{ }^{\circ} \mathrm{C}[10]$. This result is in the order of the number of rearranging units recently estimated for semicrystalline PET samples with a similar drawing ratio (but different thermal history), on the basis of Donth's approach [5]. On the other hand, for a drawing ratio of 6 , the number of rearranging units calculated with Donth's method appears to be much lower; this seems not to agree with the expectation that upon enhancing the orientation, i.e., the configurational constraints, the small-scale cooperativity should increase.

Equation (17) seems to resolve the apparent inconsistency, since the cooperativity estimated by Donth's method would correspond to $N_{\text {ind }}$. Note that in these oriented PET samples, the temperature dependence of the segmental relaxation is markedly Arrhenian [5], pointing to a local character of the process. Low values of $N_{\text {ind }}$ are expected on the basis of energetic arguments (cf. the end of Sec. III A); incidentally, this result can be formally obtained from Eq. (11) by holding $\Delta c_{p}$ to a finite value, while letting $T_{K}$ virtually approach zero in the expression of $s_{c}$.

\section{PDMS confined in nanopores}

Small-scale cooperativity in confined PDMS has been discussed to a certain extent in Ref. [6]; the effects on the large-scale rearrangements, instead, are illustrated here by comparing the relaxation behavior in different confinement regimes at a single temperature of $\sim 158 \mathrm{~K}$. For bulk PDMS, $T_{g} \simeq 150 \mathrm{~K}$ and progressively lowers upon decreasing $d_{\text {pore }}$; hence, at $T \simeq 158 \mathrm{~K}$ the expected CRR sizes will always be smaller than the actual maximum allowed.

As shown in Ref. [8], confinement affects significantly the $T$ dependence of the central relaxation rate; correspondingly, also the Vogel temperature changes. Fittings to the data of Ref. [8] yield $T_{\mathrm{VFT}}=126.5,120.3$, and $126.2 \mathrm{~K}$ for bulk, $20 \mathrm{~nm}$, and $7.5 \mathrm{~nm}$ confined samples, respectively. The last value of the Vogel temperature does not seem to be trustworthy; indeed, it is expected that the CRR size should decrease upon decreasing $d_{\text {pore }}$, and in order to compensate for the apparent trend of $T_{\mathrm{VFT}}$ in passing from 20 to $7.5 \mathrm{~nm}$ (assuming our prescriptions for the $s_{c}$ estimates are still valid), one should consider a value of $\Delta c_{p}$ larger than in bulk. For this reason, we shall take for the calculations the Vogel temperatures
TABLE III. Small- and large-scale cooperativities, $z$ and $N_{\alpha}$, ratio $\lambda / z$, temperature fluctuation $\Delta T$, and (approximate) characteristic cooperativity length $\xi$ at a temperature of $158 \mathrm{~K}$ for PDMS in different confinement regimes. The adopted Vogel temperatures $T_{\mathrm{VFT}}$ are listed as well. Values labeled with an asterisk have been calculated with $\Delta c_{p} / k_{B}=3.0$. [The $z$ values of the 20 and $7.5 \mathrm{~nm}$ samples are slightly larger than those of Ref. [6] because in the present case the analysis has been limited to time domains where $\left.\phi(t) \gtrsim 10^{-2}\right]$.

\begin{tabular}{llccccc}
\hline \hline $\begin{array}{l}\text { Confinement } \\
\text { condition }\end{array}$ & $\begin{array}{c}T_{\mathrm{VFT}} \\
(\mathrm{K})\end{array}$ & $z$ & $\lambda / z$ & $N_{\alpha}$ & $\begin{array}{c}\Delta T \\
(\mathrm{~K})\end{array}$ & $\begin{array}{c}\xi \\
(\mathrm{nm})\end{array}$ \\
\hline bulk & 130 & 2.4 & 5.1 & 38 & 3.7 & $\sim 1.7$ \\
$20 \mathrm{~nm}$ & 122.5 & 1.9 & 1.6 & 17 & 8.1 & $\sim 1.3$ \\
$7.5 \mathrm{~nm}$ & 116 & 2.5 & 1.4 & 21 & 7.3 & $\sim 1.4$ \\
$7.5 \mathrm{~nm}$ & 116 & 2.5 & 1.4 & $24^{*}$ & $6.2^{*}$ & $\sim 1.4^{*}$ \\
\hline \hline
\end{tabular}

derived under the condition $\tau_{\infty}=10^{-14} \mathrm{~s}$, namely $130,122.5$, and $116 \mathrm{~K}$ for bulk, $20 \mathrm{~nm}$, and $7.5 \mathrm{~nm}$ confined PDMS, respectively.

The value to take for $\Delta c_{p}$ at the actual $T_{g}$ represents another source of uncertainty. The work of Ref. [8] reveals that confinement depletes the average $\Delta c_{p}$ and suggests that low mobility regions form, although it is not known to what extent this lack of mobility involves the domains where the observed polarization fluctuations are localized. Certainly, the constraints induced by the low-mobility regions are most effective in $5 \mathrm{~nm}$ confinement, because in this case just the small-scale rearrangement seems to be allowed (cf. the Introduction). With the lack of further information, the calculations are performed considering for $\Delta c_{p} / k_{B}$ the bulk value of $\sim 3.4$, and the results are listed in Table III. One has to keep in mind that a decrease of $\Delta c_{p}$ would lead to larger CRRs and correspondingly smaller temperature fluctuations (an example is given in the table for completeness).

Consistent with the picture provided above by the canonical model, a slight increase of $z$ upon decreasing the pore size is indicative of an enhancement of constraints (the decrease of $z$ from bulk to $20 \mathrm{~nm}$ confinement cannot be commented upon presently, but it might be related to the observed acceleration of the dynamics [8]). On the other hand, the extent to which confinement itself is effective seems to be highlighted not only by the symmetrization of the $\alpha$-relaxation frequency profile (as usually observed), but also by the decrease of the ratio $\lambda / z$.

Although the CRR sizes reported in Table III seem reasonable, there is lingering doubt due to the lack of information about $\Delta c_{p}$. In this respect, direct measurement of the temperature fluctuations might be worthwhile.

Figure 3 shows a comparison between the $T$ dependences of bulk and $7.5 \mathrm{~nm}$ confined PDMS (from the data of Ref. [8]) with regard to $\xi$ and $\Delta T$.

\section{Atactic polystyrene}

An interesting study of the cooperativity behavior in atactic polystyrene (a-PS) was recently carried out by means of broadband heat capacity spectroscopy [21]. This material does not crystallize, and it has a glass transition temperature of about $370 \mathrm{~K}$. The characteristic length $\xi$, derived by means of Donth's method, varies from $\sim 4 \mathrm{~nm}$ at $T \approx T_{g}$ down to 


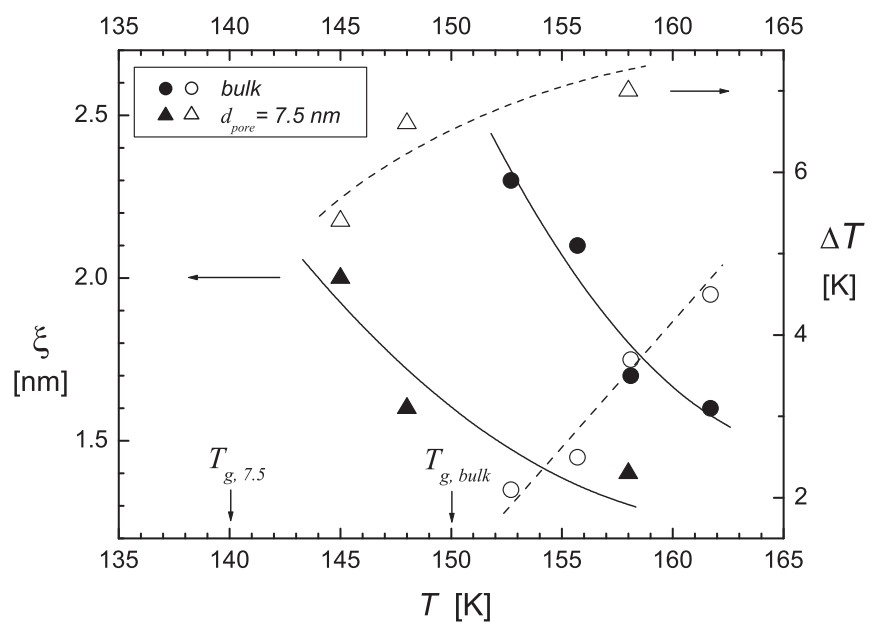

FIG. 3. Temperature dependencies of $\xi$ (filled symbols) and $\Delta T$ (open symbols) for the bulk (circles) and $7.5 \mathrm{~nm}$ confined (triangles) PDMS derived by the analysis of the data of Ref. [8]. The lines are guides for the eye. The approximate values of the calorimetric glass transition temperatures, $T_{g}$, bulk and $T_{g, 7.5}$, are indicated by the vertical arrows.

$\sim 0.7 \mathrm{~nm}$ at $T=480 \mathrm{~K}$; correspondingly, the amplitude of the temperature fluctuations increases from $\sim 4 \mathrm{~K}$ to more than $15 \mathrm{~K}$.

To provide cooperativity estimates with our method, we resort to the dielectric relaxation data of Ref. [22], which have been collected isothermally at various temperatures in the $20-10^{6} \mathrm{~Hz}$ frequency range. The study reported in that paper was carried out on a series of a-PS films of varying thickness, with the aim of investigating the effects of confinement (the effects of constraints present at the interface with the support were relevant for the thinnest films). The central relaxation times were found to follow a unique VFT line independent of the sample thickness, from hundreds of $\mu \mathrm{m}$ down to $33 \mathrm{~nm}$. Dramatic changes with regard to both an acceleration of the motion and the Vogel temperature were found, instead, for the $14 \mathrm{~nm}$ film.

Comparison with the results of Ref. [21] is done below for temperatures of 380,396 , and $414 \mathrm{~K}$. The relaxation profile of the $33 \mathrm{~nm}$ film is analyzed for the lowest $T$ value, while the response of the 194- $\mu$ m-thick sample (taken as bulk) is considered for the others.

A specific-heat step of $\Delta c_{p} \approx 5 k_{B}$ at $T_{g}$ is provided by Ref. [21]; concerning instead the Vogel temperature, two sets of VFT parameters are given in that paper, depending on whether they are derived from dielectric or calorimetric data. For consistency, we take those worked out from dielectrics, namely $\tau_{\infty}=3.16 \times 10^{-11} \mathrm{~s}, D \simeq 3.27$, and $T_{\mathrm{VFT}}=334.4 \mathrm{~K}$ (note, however, that Ref. [18] reports $\tau_{\infty}=10^{-14} \mathrm{~s}, D \simeq 4.78$, and $T_{\mathrm{VFT}} \simeq 332 \mathrm{~K}$ from viscoelastic shear compliance data).

The VFT plot reported in Ref. [22] displays the common $\tau(T)$ behavior shared by the majority of the samples, only in the interval $2.4 \times 10^{-3} \leqslant T^{-1} \leqslant 2.57 \times 10^{-3} \mathrm{~K}^{-1}$; extrapolation yields $\tau \simeq 6.7 \times 10^{-2}$ s at $T=380 \mathrm{~K}$. As shown in the inset of Fig. 4, however, there is a poor matching between the VFT of Ref. [22] and that given by [21]; in the latter case, the extrapolated $\tau$ would correspond to a temperature of $\sim 385 \mathrm{~K}$.

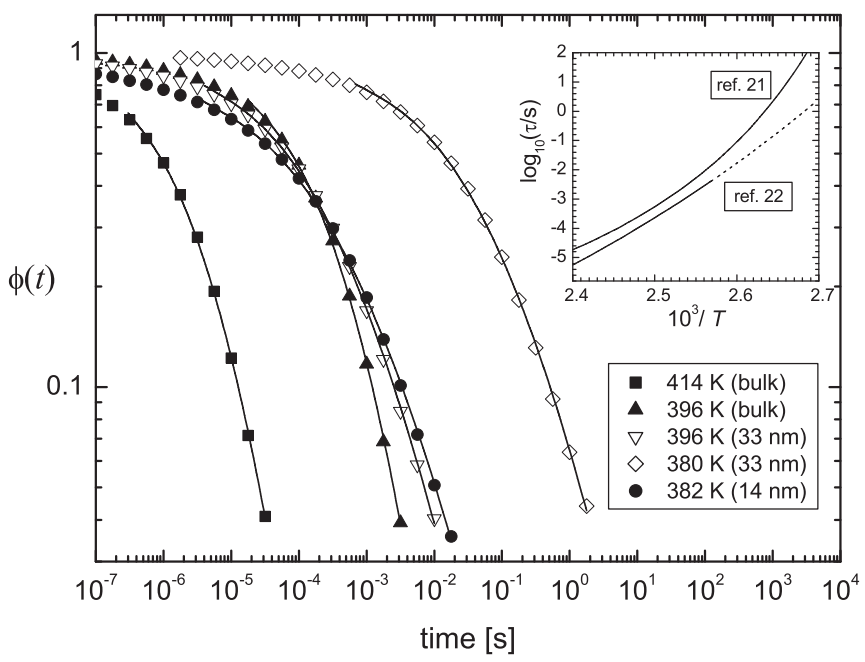

FIG. 4. Relaxation functions (symbols) corresponding to the parameters of Ref. [22] considered to work out the results of Table IV and fittings with Eq. (5) (solid lines). The inset shows the VFT lines of Refs. [21] (solid) and [22] (solid and dashed); the dashed part is an extrapolation out of the interval represented in [22].

The relative changes in $\Delta T$ and $\xi$ caused by a mismatching of just $5 \mathrm{~K}$ do not exceed $\sim 10 \%$, and this is acceptable since we are still not too close to $T_{g}$.

From the HN parameters listed in [22] and the relaxation times $\tau(T)$, the functions $\phi_{\mathrm{HN}}(t)$ can be derived, and their analysis yields the data collected in Table IV. The corresponding results of Ref. [21], reported in the same table, allow for a direct comparison.

Figure 4 shows the fittings to the functions $\phi_{\mathrm{HN}}$ yielding the results of Table IV. Note the remarkable acceleration of the dynamics in the $14 \mathrm{~nm}$ sample with respect to the case of the $33 \mathrm{~nm}$ sample at a similar temperature.

TABLE IV. Small- and large-scale cooperativities, $z$ and $N_{\alpha}$, ratio $\lambda / z$, temperature fluctuation $\Delta T$, and characteristic cooperativity length $\xi$ for a-PS at temperature $T$ (approximated to the nearest integer), worked out from the dielectric relaxation data of Ref. [22]. The last two columns on the right list, at the corresponding temperature, the approximate bounds for the observed $\Delta T$ values and the estimated characteristic lengths reported in Ref. [21]. Numbers labeled with an asterisk are derived from the relaxation data of a 33-nm-thick sample; the others (except in last row) are from a sample of $194 \mu \mathrm{m}$ thickness, which can be considered as bulk. The bottom row shows the results from a 14-nm-thick sample; in this case, $N_{\alpha}, \Delta T$, and $\xi$ are obtained assuming for $\Delta c_{p}$ the same value as bulk in the region where the cooperative motion takes place and $T_{K}=292.5 \mathrm{~K}$.

\begin{tabular}{lccccccc}
\hline \hline$T$ & & & & $\Delta T$ & $\xi$ & $\Delta T$ (Ref. [21]) & $\xi($ Ref. [21]) \\
$(\mathrm{K})$ & $z$ & $\lambda / z$ & $N_{\alpha}$ & $(\mathrm{K})$ & $(\mathrm{nm})$ & $(\mathrm{K})$ & \\
\hline 380 & $5.7^{*}$ & 2 & $130^{*}$ & $5^{*}$ & $2.7^{*}$ & $3 / 4.5$ & $2.5 / 3$ \\
396 & $4.8^{*}$ & 1.7 & $62^{*}$ & $8.8^{*}$ & $2.1^{*}$ & $6.5 / 7.5$ & 1.6 \\
& 4.3 & 3.2 & 50 & 7 & 2 & & \\
414 & 3.7 & 2.9 & 21 & 11.5 & 1.5 & $8 / 12$ & 1.3 \\
382 & 6.4 & 1.9 & 14 & 14.6 & 1.3 & & \\
\hline \hline
\end{tabular}


To check for possible influences of confinement, the results for the $33 \mathrm{~nm}$ film at $T=396 \mathrm{~K}$ and for the $14 \mathrm{~nm}$ film at $T=382 \mathrm{~K}$ have also been reported in Table IV.

The Vogel temperature of the $14 \mathrm{~nm}$ sample has been derived by fitting the data plotted in Ref. [22], yielding $T_{\mathrm{VFT}}=292.5 \mathrm{~K}$ (with $\tau_{\infty}=1.88 \times 10^{-14} \mathrm{~s}$ and $D=7.66$ ). With regard to the $c_{p}$ step, the bulk value is assumed, as in the previous case of PDMS. For this reason, the resulting values of $\Delta T$ and $\xi$ are merely qualitative, but the small-scale parameters $z$ and $\lambda$ are trustworthy. In particular, note that $\lambda / z \sim 3$ for bulk, while it decreases to about 2 for thin films. Considering the case of the $33 \mathrm{~nm}$ sample, these results suggest that the ratio $\lambda / z$ is more sensitive to confinement than is the VFT behavior.

We conclude by noting that in this system, at variance from many others, such as semicrystalline PET or nanoconfined PDMS considered above, confinement does not seem to symmetrize the frequency profile (incidentally, this feature can be seen to be also shared by semicrystalline isotactic polystyrene [23]).

\section{E. Stress relaxation on poly( $n$-butyl methacrylate) (PnBMA)}

In this case, the response of the material is directly collected in the time domain through the stress relaxation curves obtained by means of an atomic force microscope (AFM) setup. Part of the experimental details and a preliminary account of a restricted set of data can be found in Ref. [20].

\section{Experiment}

PnBMA is an amorphous polymer with a glass transition at $T_{g} \simeq 298 \mathrm{~K}$. The relaxation curves were obtained by applying a fast ramp to the $Z$-movement of a piezoelement ( $Z$-speed $>10 \mu \mathrm{m} / \mathrm{s}$ ) to increase the force from zero to a set-point value. The initial situation was that of the tip in contact with the surface with the lowest attainable stable force. After reaching the set point, the movement of the piezoelement was stopped and the relaxation of the force with time, as measured by the cantilever deflection, was acquired by a digital oscilloscope. The schematic of such an experiment is illustrated in Fig. 5(a). To compensate for the absence of a feedback mechanism on the piezo $Z$-position, the behavior related to creep movements, as measured on rigid surfaces (silicon oxide) under the same conditions, was subtracted from the relaxation curves. By exploiting the sample indentation values as a function of the force, we verified that the overall variation of the indentation during the relaxation curve was less than $11 \%$ [see Fig. 5(b)]. The cantilever used for the relaxation experiments had a spring constant of $40 \mathrm{~N} / \mathrm{m}$ (measured by the thermal method), and the tip apex (silicon) was smoothed by focused ion beam milling; the mean curvature radius of the tip was 2-3 $\mu \mathrm{m}$. All the reported measurements were obtained with the sample immersed in distilled water to avoid adhesion between the tip and the sample. The absence of adhesion was verified by AFM force curves, as shown in Fig. 6.

\section{Results}

The stress relaxation patterns, recorded for $298.6 \leqslant T \leqslant$ $312.2 \mathrm{~K}$, were first fitted with a stretched exponential function,

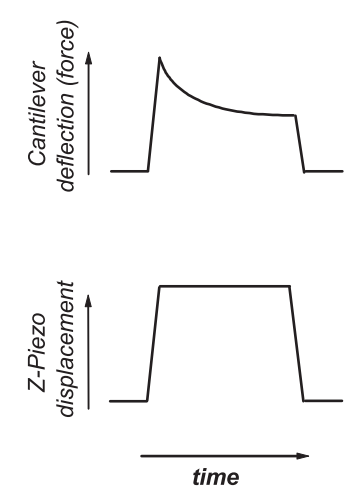

(a)

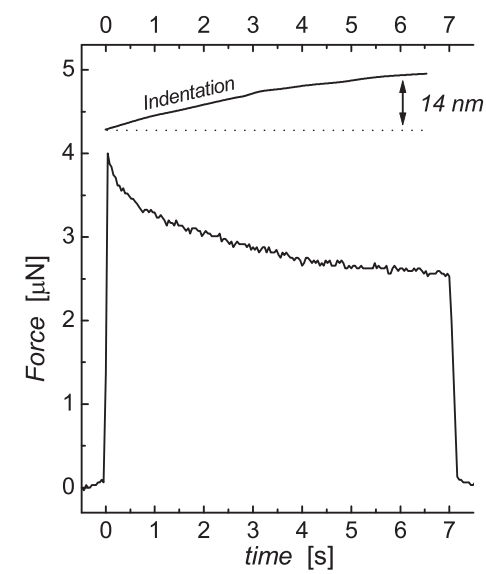

(b)
FIG. 5. Schematic representation of a force relaxation experiment performed by AFM. (a) Scheme of the Z-piezo movement (lower plot) and of the corresponding cantilever deflection (upper plot) as a function of time. The cantilever deflection is converted to the applied force by considering the force constant of the cantilever. A step toward the $Z$-piezo displacement is applied and, after reaching a maximum value, the force applied by the tip decreases with time. (b) Example of a force relaxation pattern obtained with a cantilever having a spring constant of $40 \mathrm{~N} / \mathrm{m}$ whose integrated tip has been rounded by focused ion beam milling to decrease the applied pressure and prevent plastic deformations of the polymer. The upper curve represents the corresponding indentation as a function of time.

$\phi_{\mathrm{KWW}}(t) \sim \exp \left\{-\left(t / \tau_{\mathrm{KWw}}\right)^{\beta}\right\}$, to extract the average relaxation time $\tau=\tau_{\mathrm{Kww}} \beta^{-1} \Gamma\left(\beta^{-1}\right)$. Upon lowering $T, \beta$ was found to decrease from 0.65 down to 0.55 . The VFT plot resulting from this first analysis is reported as an inset to Fig. 7.

To find CRR sizes and $\Delta T$ 's, the function $\phi(t)$ [cf. Eq. (5)] was fitted to the stretched exponentials obtained before, within time intervals where $\phi_{\mathrm{KWw}} \geqslant 0.1$. For the calculation of the

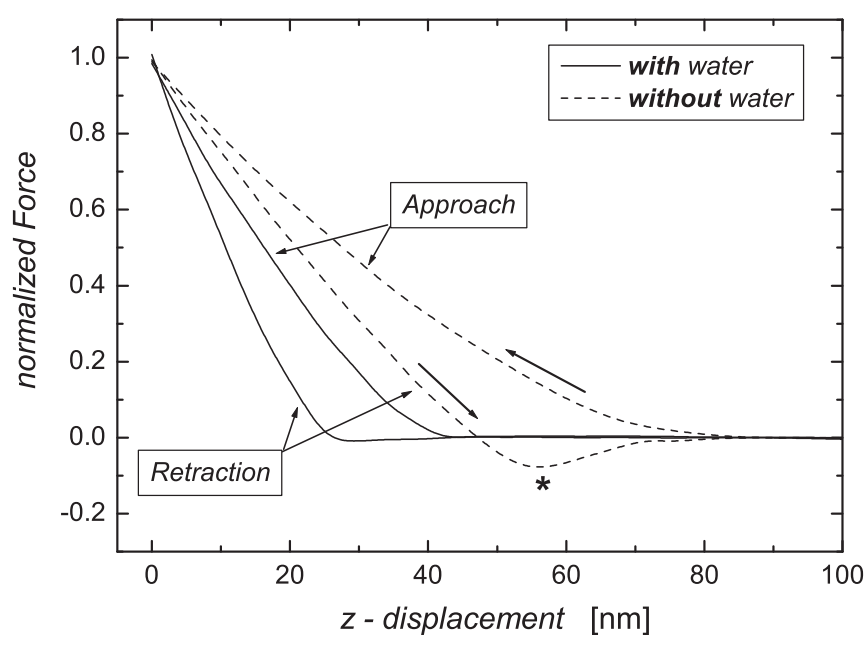

FIG. 6. Comparison between typical approach-retraction force curves in the presence or absence of water. Adhesion is pointed out by the negative force values revealed in the retraction curve $(*)$ collected in the absence of water. 


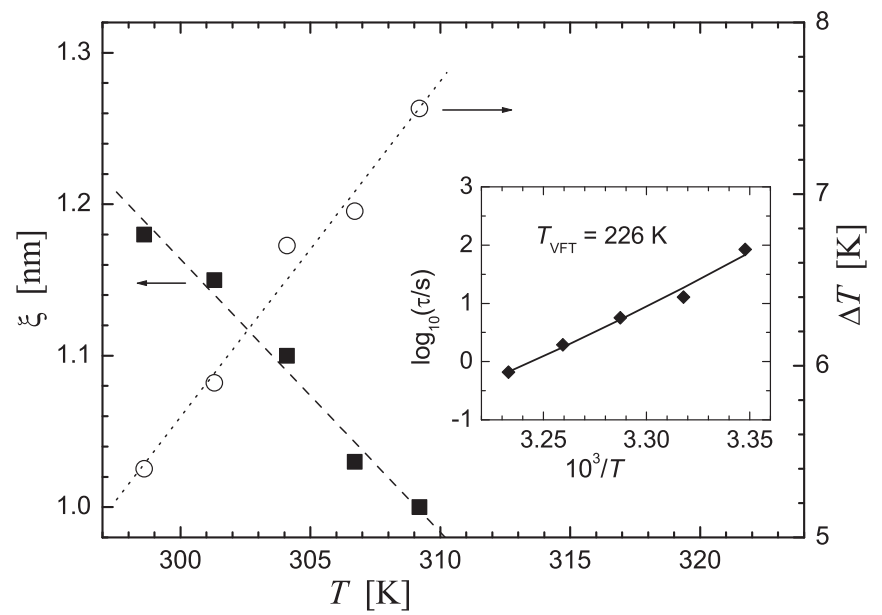

FIG. 7. Temperature dependencies of CRR size and temperature fluctuations, $\xi$ (squares) and $\Delta T$ (circles), respectively, for a sample of PnBMA $\left(T_{g} \simeq 298 \mathrm{~K}\right)$. The dashed and dotted lines are linear fits, but they only serve as guides for the eye. The inset shows the VFT behavior; the Vogel temperature $T_{\mathrm{VFT}} \simeq 226 \mathrm{~K}$ was found by fitting with Eq. (7) and setting $\tau_{\infty}$ to $10^{-14} \mathrm{~s}$.

configurational entropy, the value of $\Delta c_{p}$ at $T_{g}$ was taken from Ref. [24], while the Vogel temperature has been estimated, as before, by fitting $\tau(T)$ under the constraint $\tau_{\infty}=10^{-14} \mathrm{~s}$. The results are resumed in Fig. 7; note that the lowest- $T$ values of $\xi$ and $\Delta T$ match fairly well with those found in [2,24], namely $1 \lesssim \xi \lesssim 1.3 \mathrm{~nm}$ and $5.5 \lesssim \Delta T \lesssim 7.5 \mathrm{~K}$ at $T_{g}$.

\section{CONCLUDING REMARKS}

The approach suggested here for the estimate of the large-scale cooperativity length differs significantly from that proposed by Donth. In the latter, entropy or temperature fluctuations are directly accessed experimentally, and the characteristic length of cooperativity is calculated. On the other hand, the same quantity is estimated here on the basis of different data sets; indeed, the analysis of the relaxation focuses on the (local) activation process precursory to the large-scale configurational change and resolved in either frequency or time for a fixed temperature. The mutual theoretical consistency of the two schemes explains why such different methods of analysis provide so similar results, at least when configurational constraints do not dominate.

It is of course necessary to make improvements to the model. However, one point should be considered in particular, namely the need to provide an expression for $\tau^{*}$ disenthralling it from the mere role of a fitting parameter. It may appear indeed that large-scale cooperativity is just a by-product of small-scale cooperativity. On the contrary, $\tau^{*}$ itself appears to be sensitive to the characteristic of the large-scale segmental motion (i.e., either local or glassy). On rather naïve grounds, this idea is instilled by Eqs. (21) and (7) (considering a Taylor expansion of $s_{c}$ ). On a more concrete basis, this is suggested by the analysis of small-scale cooperativity performed on confined PDMS [6], where it was found that the $T$ dependence of $\tau^{*}$ turns from weak to strong in passing from a local $\left(d_{\text {pore }}=5 \mathrm{~nm}\right)$ to a glassy regime (e.g., with $\left.d_{\text {pore }}=7.5 \mathrm{~nm}\right)$.

A nontrivial dependence of $\tau^{*}$ on both $\overline{\Delta \mu}$ and $s_{c}$, in order to account for configurational and/or dynamical constraints (see above), is thus expected. Such a dependence should also be central to the understanding of the mechanisms involved wherever an acceleration of the relaxation dynamics upon confinement is observed.

\section{ACKNOWLEDGMENTS}

We thank Dr. Claudia Menozzi for providing us with the focused ion beam milled AFM tips we used in the stress relaxation experiment. M.P. gratefully acknowledges Ministerio de Educación y Ciencia, Spain, for the award of a sabbatical grant (SAB-2006-0077) to work at IEM, CSIC, Madrid, where the foundations of this work were put in place, and CNR, Italy, for supporting this initiative within the framework of "Congedi per Motivi di Studio."
[1] G. Adam and J. H. Gibbs, J. Chem. Phys. 43, 139 (1965).

[2] E. Donth, J. Polym. Sci. B, Polym. Phys. 34, 2881 (1996).

[3] E. Donth, The Glass Transition: Relaxation Dynamics in Liquids and Disordered Materials, Springer Series in Materials Science Vol. 48 (Springer, Berlin, 2001).

[4] C. Dalle-Ferrier, C. Thibierge, C. Alba-Simionesco, L. Berthier, G. Biroli, J.-P. Bouchaud, F. Ladieu, D. L'Hôte, and G. Tarjus, Phys. Rev. E 76, 041510 (2007).

[5] F. Hamonic, D. Prevosto, E. Dargent, and A. Saiter, Polymer 55, 2882 (2014).

[6] P. Calandra, S. Sturniolo, and M. Pieruccini, Therm. Acta 522, 135 (2011).

[7] M. Pieruccini and A. Flores, Coll. Polym. Sci. 288, 365 (2010).

[8] A. Schönhals, H. Goering, Ch. Schick, B. Frick, and R. Zorn, J. Non-Cryst. Solids 351, 2668 (2005).

[9] A. Schönhals, H. Goering, Ch. Schick, B. Frick, and R. Zorn, Eur. Phys. J. E 12, 173 (2003).
[10] M. Pieruccini and T. A. Ezquerra, Eur. Phys. J. E 29, 163 (2009).

[11] V. Lubchenko and P. G. Wolynes, J. Chem. Phys. 121, 2852 (2004).

[12] J.-P. Bouchaud and G. Biroli, J. Chem. Phys. 121, 7347 (2004).

[13] W. Greiner, L. Neise, and H. Stocker, Thermodynamics and Statistical Mechanics (Springer-Verlag, Berlin, 1995).

[14] A. Bartolotta, G. Di Marco, F. Farsaci, M. Lanza, and M. Pieruccini, Polymer 44, 5771 (2003).

[15] M. Pieruccini, A. Flores, U. Nöchel, G. Di Marco, N. Stribeck, and F. J. Baltá-Calleja, Eur. Phys. J. E 27, 365 (2008).

[16] C. Alvarez, I. Šics, A. Nogales, Z. Denchev, S. S. Funari, and T. A. Ezquerra, Polymer 45, 3953 (2004).

[17] A. Flores, F. J. Baltá-Calleja, G. Di Marco, S. Sturniolo, and M. Pieruccini, Polymer 52, 3155 (2011).

[18] B. Böhmer, K. L. Ngai, C. A. Angell, and D. J. Plazek, J. Chem. Phys. 99, 4201 (1993). 
[19] L. D. Landau and E. M. Lifšits, Fisica Statistica (Editori Riuniti, Roma, 1978), Part I.

[20] M. Pieruccini, A. Alessandrini, S. Sturniolo, M. Corti, and A. Rigamonti, Polym. Int., doi: 10.1002/pi.4894.

[21] Y. Z. Chua, G. Schulz, E. Shoifet, H. Huth, R. Zorn, J. W. P. Schmelzer, and C. Schick, Coll. Polym. Sci. 292, 1893 (2014).
[22] K. Fukao and Y. Miyamoto, Phys. Rev. E 61, 1743 (2000).

[23] B. Natesan, H. Xu, B. Seyhan Ince, and P. Cebe, J. Polym. Sci. B, Polym. Phys. 42, 777 (2004).

[24] S. Kahle, J. Korus, E. Hempel, R. Unger, S. Höring, K. Schröter, and E. Donth, Macromolecules 30, 7214 (1997). 